\title{
RECONFIGURAÇÃO DE SISTEMAS DE DISTRIBUIÇÃO UTILIZANDO FLUXO DE POTÊNCIA ÓTIMO E ANÁLISE DE SENSIBILIDADE
}

\author{
Flávio V. Gomes* \\ Paulo N. Garcia*
}

José L. R. Pereira*

Marcio P. Vinagre*

\author{
Sandoval Carneiro Jr ${ }^{\dagger}$ \\ *Faculdade de Engenharia - Campus UFJF \\ CEP 36036-330 - Juiz de Fora MG \\ ${ }^{\dagger}$ COPPE - Universidade Federal do Rio de Janeiro \\ Caixa Postal 68504, Rio de Janeiro, RJ, Brasil
}

\section{RESUMO}

Este artigo apresenta uma nova metodologia de Reconfiguração de Sistemas de Distribuição (RSD) baseada na utilização do Fluxo de Potência Ótimo (FPO) no qual o status das chaves (aberto/fechado) é representado através de funções contínuas. Na metodologia proposta, todas as chaves são consideradas inicialmente fechadas e a partir dos resultados fornecidos pelo FPO, utiliza-se uma técnica heurística para determinar o próximo laço a ser quebrado através da abertura de uma chave. A lista de chaves candidatas a abertura é atualizada e o processo descrito anteriormente é repetido até que todos os laços sejam quebrados, tornando o sistema radial. Este trabalho apresenta resultados e comparações utilizandose sistemas publicados em três artigos técnicos clássicos encontrados na literatura e em um artigo prévio do autor. Resultados obtidos em sistemas reais de distribuição também serão apresentados.

PALAVRAS-CHAVE: Reconfiguração, sistemas de distribui-

\footnotetext{
Artigo submetido em 09/05/06

1a. Revisão em 27/07/06

2a. Revisão em 13/11/06

Aceito sob recomendação do Editor Associado

Prof. Denis Vinicius Coury
}

ção radiais, otimização heurística, fluxo de potência ótimo.

\begin{abstract}
This paper presents a new approach for distribution system reconfiguration (DSR) based on optimum power flow (OPF) in which the branch statuses (open/close) are represented by continuous functions. In the proposed approach, all branches are initially considered closed and from the OPF results a heuristic technique is used to determine the next loop to be broken by opening one switch. Then the list of switches that are candidates to be opened is updated and the above process is repeated until all loops are broken, making the distribution system radial. The paper includes results and comparisons on test systems utilized in three classical papers published in the technical literature, as well as in a previous paper by the authors. Results obtained on a real large scale distribution system are also presented.
\end{abstract}

KEYWORDS: Reconfiguration, radial distribution networks, heuristic optimization technique, losses, optimum power flow. 


\section{INTRODUÇÃO}

Este artigo é essencialmente uma continuação de um estudo de Reconfiguração de Sistemas de Distribuição (RSD) publicado recentemente no qual foi descrito um novo algoritmo (Gomes et alii, 2005a e Gomes et alii, 2005b).

O algoritmo utiliza uma estratégia heurística que se inicia com um sistema de distribuição totalmente malhado obtido considerando-se todas as chaves manobráveis fechadas. Em seguida as chaves são abertas sucessivamente para eliminar os laços. Nesta técnica de abertura sequencial de chaves, o critério de abertura é baseado no mínimo incremento total de perdas ativas, as quais são determinadas através da utilização de um programa de fluxo de potência. Um refinamento ao procedimento descrito acima é obtido através da utilização de uma técnica de permuta de chaves (Peponis e Papadopoulos, 1995) envolvendo a vizinhança das chaves abertas.

Uma revisão da literatura técnica descrita em Gomes et alii, (2005a) mostra que os métodos existentes (Baran e Wu, 1989; Civanlar et alii, 1988; Goswami e Basu, 1992; McDermott et alii, 1999; Shirmohammadi e Hong, 1989) podem não determinar a configurção de perdas mínimas devido ao problema em questão possuir variáveis de natureza discreta e também de natureza contínua, tornando-o muito complexo, especialmente para grandes sistemas de distribuição.

Diversos testes foram realizados utilizando o procedimento descrito em Gomes et alii, (2005a) onde configurações mais eficientes foram obtidas quando comparadas com os métodos propostos em três artigos técnicos clássicos: McDermott $e t$ alii, (1999); Shirmohammadi e Hong, (1989) e Goswami e Basu, (1992). O método proposto em Gomes et alii, (2005a) apresenta uma boa solução, uma vez que tenta encontrar uma configuração próxima do ótimo ou até mesmo ótima sem o risco de explosão combinatorial.

O presente artigo descreve desenvolvimentos que foram realizados no trabalho descrito acima. Os autores introduziram uma formulação via FPO para reduzir o número cálculos de fluxo de potência e incorporar as restrições da redes embutidas no problema de FPO. A decisão com relação ao posicionamento das chaves é realizada por uma estratégia heurística através da utilização de uma análise de sensibilidade obtida pelo FPO. O procedimento heurístico é baseado em duas estratégias principais: $(i)$ as variáveis inteiras (chaves) são representadas através de funções contínuas na formulação do FPO; (ii) o fluxo de potência é utilizado para determinar as perdas ativas do sistema e reintroduzir a natureza discreta das chaves. Detalhes do algoritmo e os testes efetuados serão descritos nas seções seguintes.

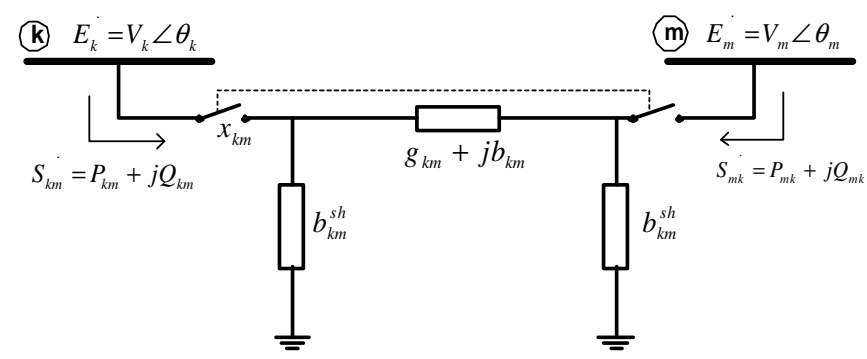

Figura 1: Modelo equivalente da linha com a chave.

\section{MODELAGEM CONTÍNUA DA CHAVE}

Na terminologia dos sistemas de distribuição as linhas são normalmente denominadas alimentadores e estes podem ser compostos de diversos ramos.

A Figura 1 ilustra a representação de um ramo e sua chave atráves de seu modelo pi-equivalente. A chave é modelada utilizando-se uma variável contínua $x_{k m}$ para a representação de sua posição, a qual assume qualquer valor entre 1 (totalmente fechada) e 0 (totalmente aberta). Estes limites serão representados no FPO através de uma restrição de canalização.

Uma chave aberta é simulada atribuindo-se um valor próximo de zero à variável $x_{k m}$, sendo este valor multiplicado pelos parâmetros correspondentes da linha (g e b). A pequena admitância resultante significa que a impedância assumirá valores elevados, logo a linha terá o comportamento de um ramo fictício. Desta forma, a rede estará sempre conexa e a matriz de impedâncias nodais não será singular.

O conceito de ramo fictício foi proposto originalmente por Monticelli (1982) na solução do problema do planejamento da expansão da transmissão. Monticelli adicionou reatâncias fictícias de elevado valor, por exemplo $10^{4}$, ao sistema original.

As chaves que assumem valor de $x_{k m}$ próximos de 1 , conforme calculados pelo FPO, indicarão que o fluxo de potência tenderá fluir naturalmente por estas linhas, permitindo uma indicação dos caminhos do fluxo. Esta é a principal razão da utilização desta idéia neste presente trabalho.

A decisão final referente à posição da chave (aberta/fechada) é tomada utilizando-se um novo procedimento baseado na valor da sensibilidade da chave fornecido pelo FPO. Virtualmente qualquer função contínua que aproxima o passo introduzido pelo status da chave poderá ser adotada. Alguns autores têm utilizado a função sigmóide (Pereira et alii, 2005) em algoritmos de expansão da transmissão obtendo ótimos resultados. No presente estudo, os autores decidiram utilizar simplesmente a equação da reta $\mathrm{y}=\mathrm{ax}(\mathrm{a}=1)$, conforme 


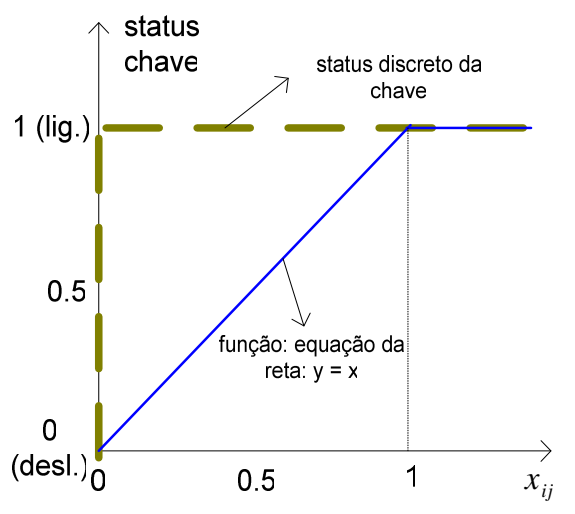

Figura 2: Função contínua utilizada para modelar a chave.

ilustrado na Figura 2.

Outros valores para o coeficiente angular podem ser adotados. Esta função foi escolhida porque todas as derivadas de segunda ordem nos elementos correspondentes da matriz Hessiana serão nulas, isto ocasionando um bom compromisso entre convergência e a qualidade dos resultados.

\section{FORMULAÇÃO FPO PROPOSTA}

O problema de RSD é convertido em um problema de otimização e pode ser formulado da seguinte forma:

$$
\begin{array}{r}
\operatorname{Min}\left\{C_{\text {total }}=\sum_{(\mathrm{k}, \mathrm{m}) \in \Omega_{l}} c_{k m}^{\text {perdas }} \cdot L_{k m}+\right. \\
\left.\sum_{(\mathrm{k}, \mathrm{m}) \in \Omega_{l}} c_{k m}^{u t i l i z} \cdot x_{k m}\right\}
\end{array}
$$

Sujeito a:

$$
\begin{gathered}
f(V, \theta)=0 \\
h(V, \theta) \leq 0 \\
0 \leq x_{k m} \leq 1 \quad \forall(\mathrm{k}, \mathrm{m}) \in \Omega_{l}
\end{gathered}
$$

onde:

$$
\begin{array}{ll}
C_{\text {total }} & \text { custo total de reconfiguração; } \\
\Omega_{l} & \text { conjunto de ramos na RSD; } \\
c_{k m}^{\text {perdas }} & \text { custo das perdas no ramo } k-m ; \\
L_{k m} & \text { perdas no ramo } k-m ; \\
c_{k m}^{u t i l i z} & \text { custo de utilização do ramo } k-m ; \\
x_{k m} & \text { variável que indica a posição da chave conec- } \\
& \text { tada entre as barras } k e m ;
\end{array}
$$

A função objetivo (1) é composta da soma de duas parcelas: (i) perdas ativas (1a): estas perdas representam custos adicionais para a empresa de distribuição, logo devem ser minimizadas;

$$
x_{k m} g_{k m}\left(V_{k}^{2}+V_{m}^{2}-2 V_{k} V_{m} \cos \theta_{k m}\right)
$$

(ii) custo de utilização da linha: esta função tem o propósito de garantir que o fluxo de potência irá fluir preferencialmente através das linhas com menores custos.

A segunda parcela acima será necessária especialmente em problemas de planejamento onde os ramos possuem diferentes custos de construção. Para o problema de RSD os ramos serão normalmente considerados com tendo um mesmo custo não nulo. Um custo nulo no FPO produziria uma solução onde todas as chaves estariam fechadas para minimizar as perdas.

A Equação (2) corresponde às equações de balanço de fluxo de potência ativa (2a) e reativa (2b) no sistema de distribuição.

$$
\begin{aligned}
& P_{k}=P_{k}^{\text {alim }}-P_{k}^{\text {carga }}-\sum_{m \in \Omega_{l}} P_{k}^{\text {linham }} \\
& Q_{k}=Q_{k}^{\text {alim }}-Q_{k}^{\text {carga }}-\sum_{m \in \Omega_{l}} Q_{k}^{\text {linham }}
\end{aligned}
$$

A Equação (3) leva em consideração os limites operacionais tais como limite de fluxo de potência ativa nas linhas. A Equação (4) considera as restrições de canalização tais como limites das tensões nas barras, limites de carga dos alimentadores, e de posicionamento das chaves. Além disso, as restrições na estrutura topológica precisam ser consideradas.

Minimizar (1) significa simultaneamente minimizar as parcelas (i) e (ii). Reduzir (i) implica em aumentar o valor da posição da chave (aproximar de 1), uma vez que em um sistema malhado as perdas tendem a se reduzir. Por outro lado, para reduzir (ii) implica em reduzir o valor da posição da chave correspondente. Logo as funções são, de certa forma, conflitantes. Todavia, uma vez que a soma é uma soma ponderada, os valores para dos custos das perdas e para o custo de utilização do ramo são baseados em uma análise de sensibilidade. No presente trabalho foram utilizados valores entre 0.001 e 0.1 para a utilização do ramo, e 1 para o custo das perdas.

Para solucionar o algoritmo de FPO, equações $(1$ - 4), foi utilizada a técnica de otimização por pontos interiores primaldual (Wright, 1997; Castronuovo et alii, 2001).

\section{DESCRIÇÃO DO MÉTODO PROPOSTO}

A Figura 3 ilustra um fluxograma do método proposto, o qual é baseado numa sequencia de seis passos. 


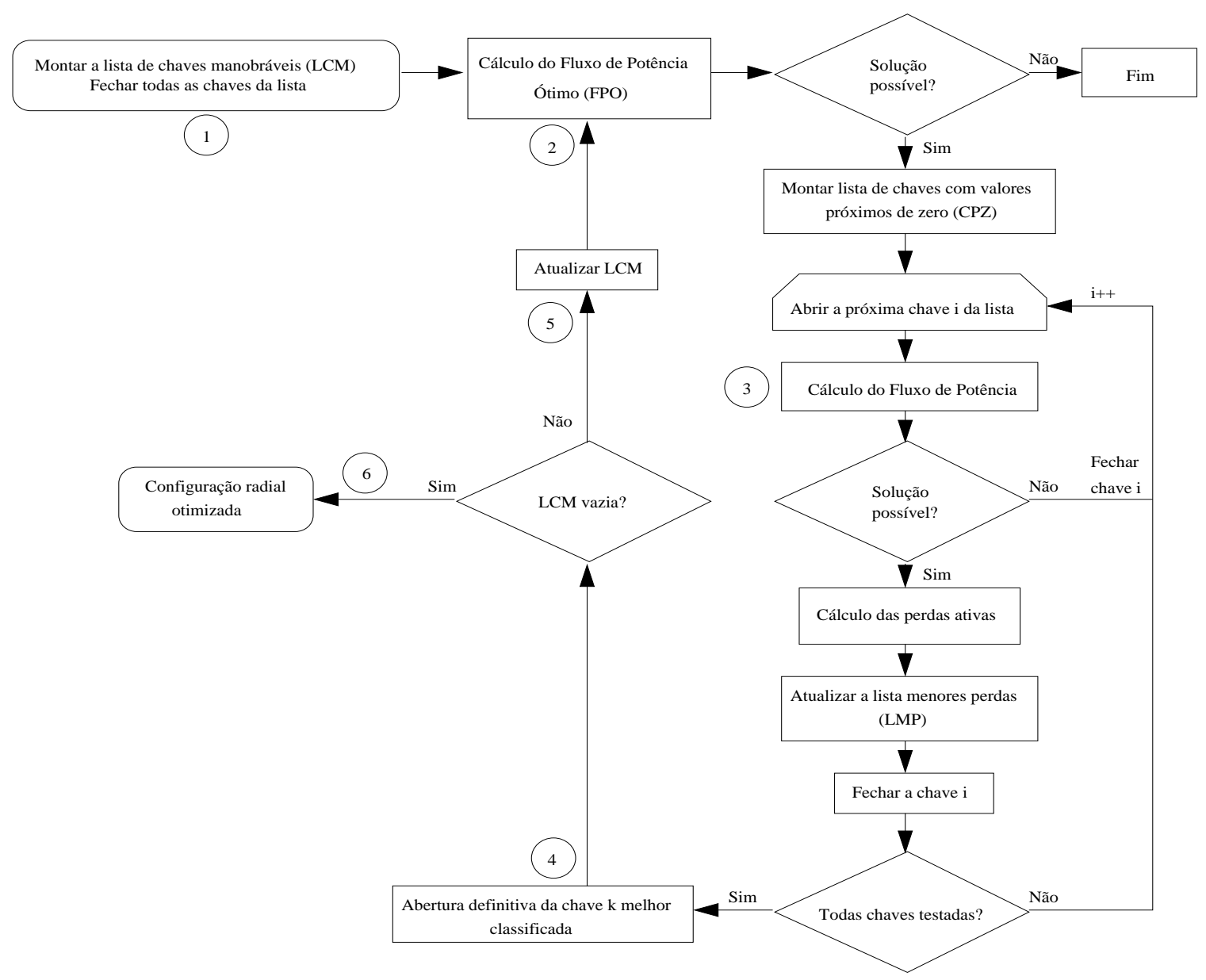

Figura 3: Algoritmo proposto para reconfiguração de sistemas de distribuição.

\section{Passo 1: Montar a lista de chaves manobráveis}

Inicialmente constrói-se uma lista de chaves manobráveis (LCM). Esta lista deve conter todas as $z$ chaves do sistema que devem ser consideradas no processo de otimização. A solução do método proposto inicia-se com uma rede totalmente malhada considerando-se todas as chaves da LCM fechadas.

\section{Passo 2: Cálculo do fluxo de potência ótimo}

O FPO fornecerá os valores de $x_{k m}$ para todas as chaves manobráveis. Com o objetivo de reduzir o número de soluções de fluxo de potência necessárias no passo 3 , um subconjunto de chaves é escolhido. Este subconjunto chamado CPZ é compreendido pelas chaves cujos valores estão próximos de zero. Experiências com o algoritmo mostraram que um conjunto com a dimensão de duas vezes o número de chaves normalmente abertas (necessárias para garantir a radialidade) é adequado.
Se a solução não é obtida (FPO não converge), as restrições devem ser analisadas, assim como os pesos nas parcelas da função objetivo.

\section{Passo 3: Cálculo do fluxo de potência}

Neste passo $n$ configurações de rede são produzidas de forma que, para cada uma delas somente uma chave $i(i<=n)$ é aberta, enquanto todas as remanescentes são mantidas fechadas. As configurações são identificadas pelo número da chave correspondente. Cada configuração $i$ é testada e se uma rede conexa é obtida (quando a chave $i$ é aberta), um fluxo de potência não linear é calculado. Se a rede não for conexa, ou se qualquer restrição for violada (limites de tensão e fluxos) a configuração é descartada e o algoritmo passa para a próxima configuração a ser analisada. Para qualquer configuração de rede $i$ válida, as perdas totais do sistema são calculadas e armazenadas numa lista por ordem crescente de perdas (LMP). Finalmente a chave $i$ é fechada e os passos 


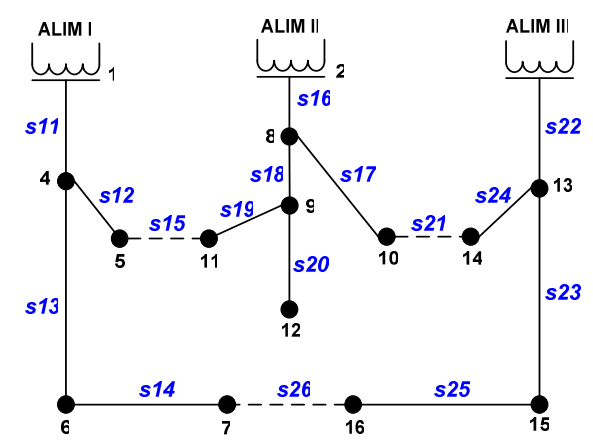

Figura 4: Exemplo 3 alimentadores: configuração inicial

acima são repetidos até que todas as $n$ configurações sejam processadas.

\section{Passo 4: Abertura definitiva da chave que produzir o me- nor incremento de perdas}

A lista de classificação das chaves segundo as perdas (LMP) é utilizada para determinar a configuração que resultou no menor incremento de perdas no sistema através da abertura da chave, consideraremos, por hipótese, a chave $k$. Logo a chave $k$ é selecionada para ser aberta definitivamente.

\section{Passo 5: Atualizar a lista de chaves manobráveis}

Após a abertura definitiva de uma determinada chave $k$, a LCM deve ser atualizada (atualizar $z$ ) de forma que a chave $k$ deve ser removida assim como todas as chaves pertencentes ao mesmo laço desfeito e que não pertencem simultaneamente a outros laços, pois suas aberturas poderiam conduzir a sistemas desconexos.

\section{Passo 6:}

O procedimento descrito anteriormente é aplicado em sequência à atualização da LCM até o ponto em que a LCM se tornar vazia, situação na qual todos os laços estarão desfeitos ou, em outras palavras, o sistema se tornar radial. Enquanto a LCM não estiver vazia, retornar ao passo (2).

\section{EXEMPLO}

A Figura 4 ilustra a configuração inicial de um sistema teste (Civanlar et alii, 1988; Whei-Min e Hong-Chan 1998), consistindo de 3 alimentadores, 13 chaves seccionadoras e 3 chaves de interligação entre alimentadores.

\section{Passo 1: Montar a lista de chaves manobráveis}

Inicialmente todas as 16 chaves manobráveis são utilizadas
Tabela 1: Posicionamento Chave e Perdas totais em Ordem Crescente $\left(1^{\circ}\right.$ Laço $)$

\begin{tabular}{|c|c|}
\hline Chave & Posição \\
\hline s26 & $9.83738 \mathrm{e}-005$ \\
\hline $\mathrm{s} 19$ & 0.000735234 \\
\hline $\mathrm{s} 21$ & 0.007907280 \\
\hline $\mathrm{s} 15$ & 0.008451880 \\
\hline $\mathrm{s} 24$ & 0.018470000 \\
\hline $\mathrm{s} 17$ & 0.018491900 \\
\hline
\end{tabular}

\begin{tabular}{|c|c|}
\hline Chave & Perdas $(\mathrm{kW})$ \\
\hline s26 & 426.47 \\
\hline s17 & 428.24 \\
\hline s21 & 443.82 \\
\hline s24 & 456.25 \\
\hline s19 & 456.97 \\
\hline s15 & 472.43 \\
\hline
\end{tabular}

no processo de otimização: s11 a s26. Um sistema de distribuição malhado é obtido considerando toda a LCM fechada.

\section{Passo 2: Cálculo do fluxo de potência ótimo}

Obtém-se o posicionamento contínuo das chaves através da utilização do FPO. Um conjunto formado pelas chaves com os 6 menores valores de posição $(\mathrm{CPZ})$ é obtido, conforme mostrado na Tabela 1

\section{Passo 3: Cálculo do fluxo de potência}

Neste passo são geradas 6 topologias, de acordo com o passo 3 descrito na seção 4 . É calculado um fluxo de potência e as perdas produzidas para cada topologia obtida. As 6 configurações obtidas são classificadas em ordem crescente das perdas, conforme ilustrado na Tabela 1.

\section{Passo 4: Abertura definitiva da chave que produzir o me- nor incremento de perdas}

Como pode ser observado através da Tabela 1, a chave s26 é a que produz as menores perdas no sistema. Portanto, neste passo, esta é a chave escolhida para ser aberta definitivamente.

\section{Passo 5: Atualizar a lista de chaves manobráveis}

Uma vez que a chave s26 é escolhida para ser aberta definitivamente, todas as chaves pertencentes ao mesmo laço e que não pertençam simultaneamente a outros laços, devem ser excluídas da LCM, caso contrário sua abertura produziria sistemas desconexos. Logo as seguintes chaves são removidas da LCM: s26, s25, s23, s14 e s13.

\section{Passo 6:}

O processo é repetido iniciando-se no passo 2 e com a LCM atualizada. Um novo conjunto de chaves com os menores valores de posição CPZ é obtido e 6 novas topologias são produzidas conforme ilustrado na Tabela 2. Percebe-se que a chave s17 é escolhida para ser a segunda chave a ser aberta definitivamente. As chaves s17, s21, s22 e s24 são excluídas da LCM. Na terceira (e última) iteração do algoritmo, um 
Tabela 2: Posicionamento Chave e Perdas totais em Ordem Crescente ( $2^{\circ}$ Laço)

\begin{tabular}{|c|c|}
\hline Chave & Posição \\
\hline s19 & 0.000704751 \\
\hline s21 & 0.008160530 \\
\hline s15 & 0.008465710 \\
\hline s24 & 0.018314500 \\
\hline s17 & 0.018423900 \\
\hline s12 & 0.055418800 \\
\hline
\end{tabular}

\begin{tabular}{|c|c|}
\hline Chave & Perdas $(\mathrm{kW})$ \\
\hline s17 & 430.03 \\
\hline s21 & 449.12 \\
\hline s19 & 455.86 \\
\hline s24 & 462.29 \\
\hline s15 & 471.98 \\
\hline s12 & 612.29 \\
\hline
\end{tabular}

Tabela 3: Posicionamento Chave e Perdas totais em Ordem Crescente ( $3^{\circ}$ Laço)

\begin{tabular}{|c|c|}
\hline Chave & Posição \\
\hline s19 & 0.000651793 \\
\hline s15 & 0.008499770 \\
\hline s12 & 0.055545400 \\
\hline s11 & 0.132017000 \\
\hline s18 & 0.148163000 \\
\hline s16 & 0.245240000 \\
\hline
\end{tabular}

\begin{tabular}{|c|c|}
\hline Chave & Perdas $(\mathrm{kW})$ \\
\hline $\mathrm{s} 19$ & 466.13 \\
\hline $\mathrm{s} 15$ & 483.87 \\
\hline $\mathrm{s} 12$ & 639.55 \\
\hline $\mathrm{s} 18$ & 705.03 \\
\hline $\mathrm{s} 16$ & 1180.74 \\
\hline $\mathrm{s} 11$ & 1270.57 \\
\hline
\end{tabular}

novo CPZ e 6 novas topologias são obtidas. Conforme ilustrado na Tabela 3, a última chave a ser aberta definitivamente é a s19.

A solução encontrada utilizando o método proposto consiste na abertura das chaves: s26, s17 and s19. Neste caso, esta é a solução ótima global. A Figura 5 ilustra a configuração final do sistema.

\section{RESULTADOS}

Nesta seção serão apresentados os resultados obtidos utilizando-se o sistema apresentado em Baran e Wu (1989) e um sistema de distribuição brasileiro.

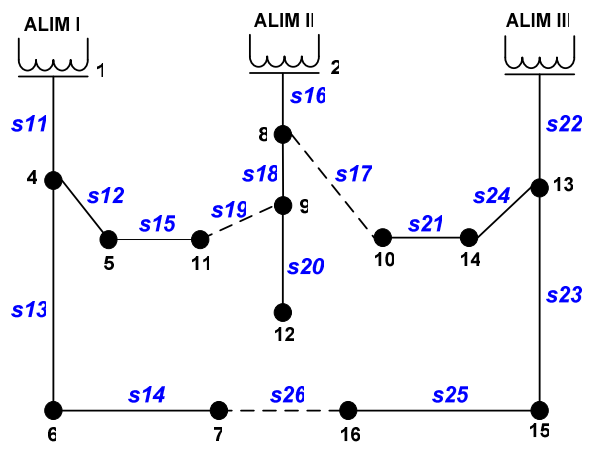

Figura 5: Exemplo 3 alimentadores: configuração final

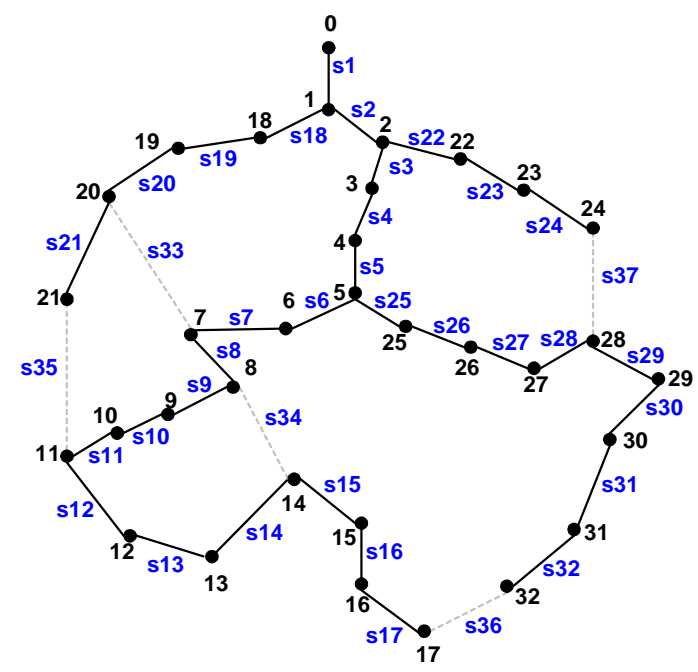

Figura 6: Sistema teste 33 barras Baran \& Wu.

O método proposto e os algoritmos descritos em Gomes et alii, (2005a); McDermott et alii, (1999); Shirmohammadi e Hong, (1989) e Goswami e Basu, (1992) foram todos implementados utilizando-se $\mathrm{C}++$ para comparação de resultados.

A metodologia proposta utiliza recursos computacionais eficientes tais como fluxo de potência ótimo via Método de Pontos Interiores Primal-Dual, rotina de fluxo de potência utilizando-se Newton-Raphson em coordenadas retangulares (Garcia et alli, 2000) e rotinas para verificação de sistemas desconexos e identificação de laços. Uma rotina de avaliação em que todas as configurações de rede possíveis são testadas também foi programada. Os tempos computacionais foram obtidos utilizando-se um Pentium 4, 2.8 Mhz, 512 MB memória RAM.

\section{Caso A. Sistema Teste Baran \& Wu}

O sistema Baran e Wu (1989) de 12.66 kV ilustrado na Figura. 6 consiste de 33 barras, 5 linhas de interligação entre alimentadores, $5058.25 \mathrm{~kW}$ e $2547.32 \mathrm{kvar}$. As chaves normalmente abertas s33, s34, s35, s36, s37 são representadas por linhas tracejadas. As chaves normalmente fechadas $\mathrm{s} 1 \mathrm{a}$ s32 são representadas por linhas sólidas. Para este caso, as perdas iniciais são $202.68 \mathrm{~kW}$.

A Tabela 4 fornece uma comparação entre diversos algoritmos. Observa-se que os métodos Gomes et alli, (2005a), McDermott et alli (1999) e Goswami e Basu (1992) encontram a configuração global ótima. Uma solução próxima do ótimo foi encontrada aplicando-se o método proposto e o algoritmo Shirmohammadi e Hong (1989). Neste caso pode-se considerar que todos os algoritmos obtiveram a solução ótima visto que os valores são muito próximos. 
Tabela 4: RESUMO DOS RESULTADOS OBTIDOS PARA DIFERENTES MÉTODOS: CASO A

\begin{tabular}{|l|c|c|c|c|}
\hline Método & $\begin{array}{c}\text { PERdAS } \\
\text { Finais } \\
(\mathbf{K W})\end{array}$ & $\begin{array}{c}\text { REdUÇão } \\
(\boldsymbol{\%})\end{array}$ & $\begin{array}{c}\text { CPU } \\
(\mathbf{S})\end{array}$ & $\begin{array}{c}\text { CHAVES } \\
\text { ABERTAS }\end{array}$ \\
\hline Ótimo* & 136.57 & 32.6 & 647.03 & s7 s9 s14 s32 s37 \\
\hline Gomes & 136.57 & 32.6 & 1.66 & s7 s9 s14 s32 s37 \\
\hline Goswami & 136.57 & 32.6 & 0.87 & s7 s9 s14 s32 s37 \\
\hline McDermott & 136.57 & 32.6 & 1.99 & s7 s9 s14 s32 s37 \\
\hline Proposto & 136.66 & 32.5 & 0.96 & s7 s10 s14 s32 s37 \\
\hline Shirmohammadi & 136.66 & 32.5 & 0.14 & s7 s10 s14 s32 s37 \\
\hline
\end{tabular}

Tabela 5: RESUMO DOS RESULTADOS OBTIDOS PARA DIFERENTES MÉTODOS: CASO B

\begin{tabular}{|c|c|c|c|c|}
\hline MÉTODO & $\begin{array}{c}\text { PERDAS } \\
\text { FINAIS } \\
(\mathrm{KW})\end{array}$ & $\begin{array}{c}\text { REDUÇÃo } \\
(\%)\end{array}$ & $\begin{array}{c}\text { CPU } \\
(\mathbf{s})\end{array}$ & $\begin{array}{c}\text { Chaves } \\
\text { Abertas }\end{array}$ \\
\hline Ótimo * & 136.57 & 30.8 & 647.03 & s7 s9 s14 s32 s37 \\
\hline Gomes & 136.57 & 30.8 & 1.66 & s7 s9 s14 s32 s37 \\
\hline McDermott & 136.57 & 30.8 & 1.99 & s7 s9 s14 s32 s37 \\
\hline Proposto & 136.66 & 30.7 & 0.96 & s7 s10 s14 s32 s37 \\
\hline Shirmohammadi & 136.66 & 30.7 & 0.14 & s7 s10 s14 s32 s37 \\
\hline Goswami & 143.71 & 27.1 & 0.65 & s11s28s32s33s34 \\
\hline
\end{tabular}

\section{Caso B. Sistema Teste Baran \& Wu Modificado}

A configuração inicial ilustrada na Figura. 6 foi alterada fechando-se as chaves normalmente abertas s33 e s37 e abrindo-se as chaves s3 e s6. As condições de carregamento foram mantidas. Para este caso as perdas iniciais são de $197.22 \mathrm{~kW}$.

Os resultados são ilustrados na Tabela 5, onde pode-se observar que os resultados obtidos com o método proposto assim como os fornecidos pelos algoritmos Gomes et alli, McDermott et alli e Shirmohammadi et alli, não sofreram alterações em relação ao Caso A. Entretanto, a configuração obtida com o método proposto por Goswami e Basu não é a mesma do Caso A, o que significa que o algorithmo depende da configuração inicial do sistema. O método proposto não depende da configuração inicial do sistema uma vez que este inicia-se com todas as chaves manobráveis fechadas.

\section{Caso C. Modificação nas Cargas das Barras 9 e 13}

Este teste foi derivado do Caso B sobrecarregando-se algumas barras do sistema. Este caso foi concebido para ilustrar a robustez do algoritmo proposto. As cargas na barra $9(60 \mathrm{~kW}$ e $20 \mathrm{kvar}$ ) e na barra 13 (120 kW and $80 \mathrm{kvar}$ ) foram ambas substituidas por $420 \mathrm{~kW}$ and $200 \mathrm{kvar}$. Para este caso as perdas na configuração inicial são de 299.94 kW.
Tabela 6: RESUMO DOS RESULTADOS OBTIDOS PARA DIFERENTES MÉTODOS: CASO C

\begin{tabular}{|l|c|c|c|l|}
\hline MÉtodo & $\begin{array}{c}\text { PERDAS } \\
\text { FinAIS } \\
(\mathbf{K W})\end{array}$ & $\begin{array}{c}\text { REDUÇão } \\
(\boldsymbol{\%})\end{array}$ & $\begin{array}{c}\text { CPU } \\
(\mathbf{S})\end{array}$ & $\begin{array}{c}\text { ChaVES } \\
\text { ABERTAS }\end{array}$ \\
\hline Ótimo * & 198.11 & 34.0 & 688.40 & $\mathrm{~s} 9 \mathrm{~s} 14 \mathrm{~s} 32 \mathrm{~s} 28 \mathrm{~s} 33$ \\
\hline Proposto & 199.56 & 33.5 & 0.99 & $\mathrm{~s} 7 \mathrm{~s} 10 \mathrm{~s} 14 \mathrm{~s} 28 \mathrm{~s} 36$ \\
\hline Gomes & 199.56 & 33.5 & 1.72 & $\mathrm{~s} 7 \mathrm{~s} 10 \mathrm{~s} 14 \mathrm{~s} 28 \mathrm{~s} 36$ \\
\hline Shirmohammadi & 201.87 & 32.7 & 0.17 & $\mathrm{~s} 7 \mathrm{~s} 10 \mathrm{~s} 14 \mathrm{~s} 17 \mathrm{~s} 37$ \\
\hline Goswami & 202.77 & 32.4 & 0.65 & $\mathrm{~s} 10 \mathrm{~s} 14 \mathrm{~s} 28 \mathrm{~s} 33 \mathrm{~s} 36$ \\
\hline McDermott & 204.89 & 31.7 & 2.14 & s7 s9 s13 s32 s37 \\
\hline
\end{tabular}

A Tabela 6 ilustra uma comparação entre os diversos algoritmos, onde pode-se observar que o método proposto fornece uma configuração que resultam numa redução de $33.5 \%$ das perdas do sistema. Nota-se que inclusive para uma péssima condição inicial com com altas perdas o algoritmo pode obter uma solução próxima do ótimo.

\section{Caso D. Sistema de Distribuição Brasileiro}

Para verificar a eficiência do método proposto em um sistema de distribuição de grande porte, o algoritmo foi aplicado a um sistema de distribuição brasileiro de $13.8 \mathrm{kV}$. A rede consiste de 2 alimentadores: o primeiro com 258 barras, carga total de $5140 \mathrm{~kW}$ e 1949 kvar. O segundo alimentador possui 218 barras, carga total de $3874 \mathrm{~kW}$ e 1498 kvar. Um total de 22 chaves seccionadoras e de interligação foram consideradas para utilização no processo de reconfiguração. Para este caso as perdas iniciais foram de $202.09 \mathrm{~kW}$ e todos os controles foram especificados em suas condições nominais. A Figura 7 ilustra um diagrama simplificado deste sistema.

Observa-se através da Tabela 7 que o método proposto obteve uma configuração de rede que resulta numa redução de $21 \%$ das perdas totais do sistema, valor que é substancialmente melhor do que as soluções obtidas pelos outros métodos, McDermott et alli, Shirmohammadi et alli e Goswami e Basu. As chaves abertas obtidas pelos vários métodos são ilustradas na Tabela 8 .

Observa-se que o tempo computacional utilizado por Shirmohammadi et alli é o menor em todos os casos uma vez que este método realiza apenas um cálculo de fluxo de potência para cada laço quebrado.

\section{CONCLUSÕES}

Este artigo descreveu uma metodologia que utiliza FPO para determinar a sensibilidade das chaves que possuem posições próximas de zero (abertas) e determinar quais chaves devem ser definitivamente abertas de forma sequencial até que o sis- 


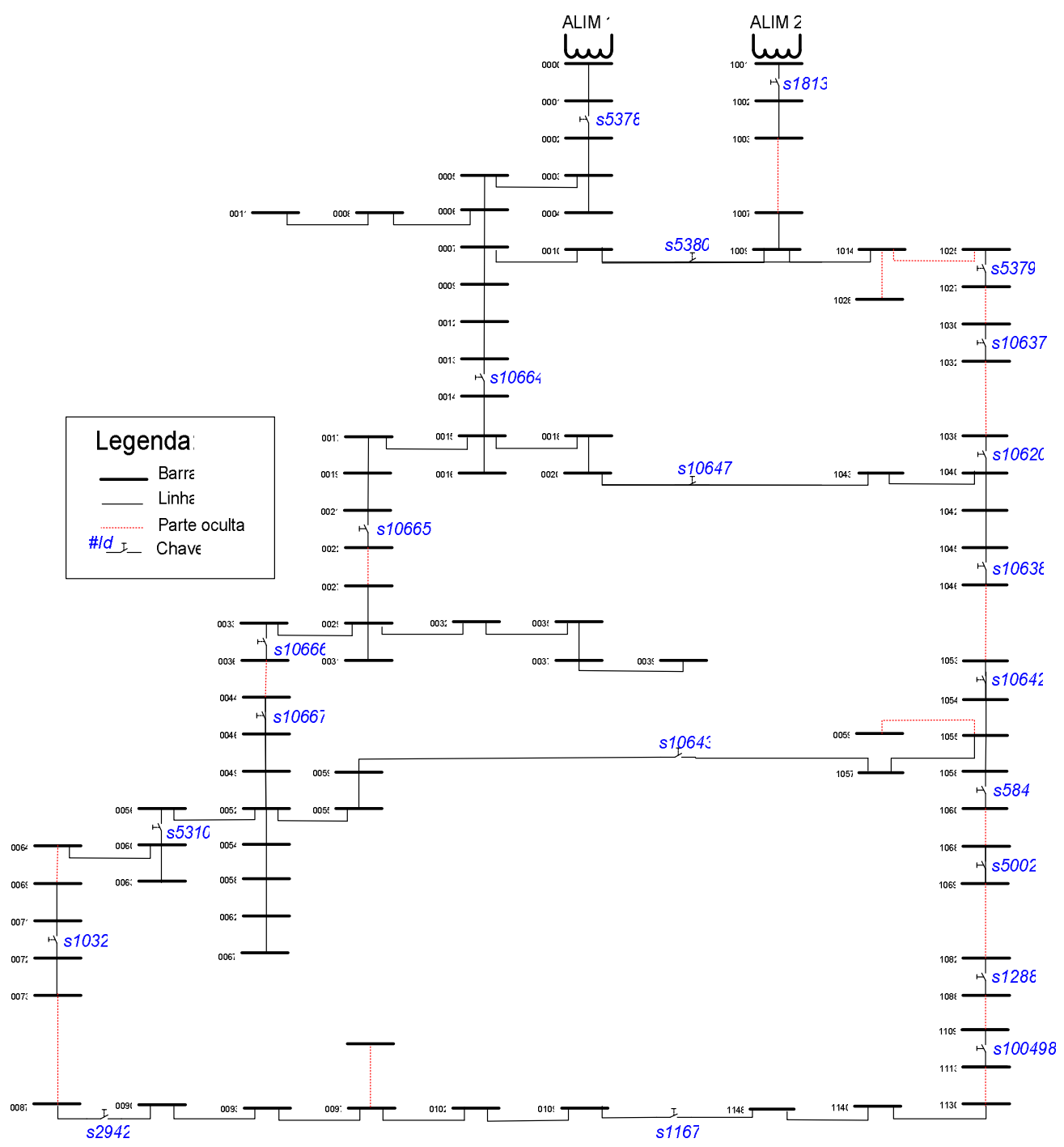

Figura 7: Sistema teste Caso D

Tabela 7: RESUMO DOS RESULTADOS OBTIDOS PARA DIFERENTES MÉTODOS: CASO D

\begin{tabular}{|l|c|c|c|}
\hline \multicolumn{1}{|c|}{ MÉtodo } & $\begin{array}{c}\text { PERDAS FINAIS } \\
(\mathbf{K W})\end{array}$ & $\begin{array}{c}\text { REDUÇão } \\
(\mathbf{\%})\end{array}$ & CPU (S) \\
\hline Ótimo * & 159.67 & 21.0 & 238.56 \\
\hline Proposto & 159.67 & 21.0 & 9.73 \\
\hline Gomes & 159.67 & 21.0 & 14.87 \\
\hline McDermott & 192.24 & 4.9 & 10.71 \\
\hline Goswami & 195.08 & 3.5 & 4.87 \\
\hline Shirmohammadi & 195.08 & 3.5 & 1.09 \\
\hline
\end{tabular}

tema se torne radial.

Diversos testes foram efetuados e os resultados obtidos mostraram que é alcançada uma solução global ótima ou muito
Tabela 8: CHAVES ABERTAS: CASO D

\begin{tabular}{|l|c|}
\hline \multicolumn{1}{|c|}{ MÉTODo } & CHAVES ABERTAS \\
\hline Ótimo * & s2942 s5380 s10643 s10647 \\
\hline Proposto & s2942 s5380 s10643 s10647 \\
\hline Gomes & s2942 s5380 s10643 s10647 \\
\hline McDermott & s2942 s5380 s10647 s10667 \\
\hline Goswami & s1167 s5380 s10647 s10667 \\
\hline Shirmohammadi & s1167 s5380 s10647 s10667 \\
\hline
\end{tabular}

* Obtidos com o algoritmo de força-bruta

próxima do ótimo. Os conjuntos solução de chaves obtidos produziram configurações de rede mais eficientes resultando em perdas menores quando comparadas com outros métodos 
disponíveis na literatura.

A incorporação da equação da reta como uma função contínua no algoritmo de FPO em associação com a técnica heurística proposta fornece ótimos resultados.

Os resultados obtidos com o aprimoramento proposto, quando comparados com o método anterior proposto pelos autores (Gomes et alii, 2005a), mostra que a introdução do algoritmo de FPO contribuiu para a redução do número de fluxos de potência e incorporou as restrições da rede. A performance do algoritmo heurístico com a utilização do FPO consistiu num aprimoramento e pode ser aplicado a sistemas reais de distribuição.

\section{REFERÊNCIAS}

Arias-Albornoz, M. e Sanhueza-Hardy, H. (2004). Distribution network configuration for minimum energy supply cost, IEEE Trans. Power Systems, vol. 19, no. 1, pp. 538-542.

Baran, M. E. e Wu, F. F. (1989). Network reconfiguration in distribution systems for loss reduction and load balancing. IEEE Trans. Power Delivery, vol. 4, no. 2, pp. $1401-1407$.

Castronuovo, E. D.; Campagnolo, J. M. e Salgado, R. (2001). On the application of high performance computation techniques to nonlinear interior point methods, IEEE Trans. Power Apparatus and Systems, v. 16, n. 3, pp. 325-331.

Ching-Tzong, S. e Lee, C. S. (2003). Network reconfiguration of distribution systems using improved mixedinteger hybrid differential evolution, IEEE Trans. Power Delivery, vol. 18, no. 3, pp. 1022-1027.

Civanlar, S.; Grainger, J. J.; Yin, H. e Lee S. S. H. (1988). Distribution feeder reconfiguration for loss reduction, IEEE Trans. Power Delivery, vol. 3, no. 3, pp. $1217-1223$.

Garcia, P. A. N.; Pereira, J. L. R.; Carneiro, S.; Vinagre, M. P. e Gomes, F. V. (2000). Improvements in the representation of PV buses on three-phase distribution power flow. IEEE Trans. Power Delivery, vol.19, no. 24, pp. $894-896$.

Gomes, F. V.; Carneiro, S.; Pereira, J. L. R; Vinagre, M. P. e Garcia P. A. N. (2005a). A new heuristic reconfiguration algorithm for large distribution systems, IEEE Trans. Power Systems, vol. 20, no. 3, pp. 1373-1378.

Gomes, F. V.; Carneiro, S.; Pereira, J. L. R; Vinagre, M. P. e Garcia P. A. N. (2005b). A new distribution system re- configuration approach using optimal power flow technique and sensitivity analysis for loss reduction, Power Engineering Society General Meeting, 2005, vol. 1, pp. 897-901.

Goswami, S. K. e Basu, S. K. (1992). A new algorithm for the reconfiguration of distribution feeders for loss minimization, IEEE Trans. Power Delivery, vol. 7, no. 3, pp. 1484-1491.

Hsiao, Y. T. (2004). Multiobjective evolution programming method for feeder reconfiguration, IEEE Trans. Power Systems, vol. 19, no. 1, pp. 594-599.

López, E.; Opazo, H.; García, L. e Bastard P. (2004). Online reconfiguration considering variability demand: applications to real networks, IEEE Trans. Power Systems, vol. 19 , no. 1, pp. 549-553.

McDermott, T. E.; Drezga, I. e Broadwate R. P. (1999). A heuristic nonlinear constructive method for distribution system reconfiguration, IEEE Trans. Power Systems, vol. 14 , no. 2 , pp. $478-483$.

Monticelli, A. (1982). Interactive transmission network planning using a least-effort criterion, IEEE Trans. Power Apparatus and Systems, v. 101, n. 10, pp. 3919-3925.

Peponis, G. e Papadopoulos M. (1995). Reconfiguration of radial distribution networks: application of heuristic methods on large-scale networks, IEE Proc-Gener. Transm. Distribution, vol. 142, no. 6, pp. 631-638.

Pereira, J. L. R.; Silva, I. C.; Oliveira, E. J. e Carneiro, S. (2005). Transmission system expansion planning using a sigmoid function to handle integer investment variables, IEEE Trans. Power Systems, vol.. 20, no. 3, pp. 1616-1620.

Shirmohammadi, D. e Hong H. W. (1989). Reconfiguration of electric distribution for resistive line loss reduction, IEEE Trans. Power Delivery, vol. 4, no. 2, pp. $1492-1498$.

Venkatesh, B.; Ranjan, R. e Gooi H. B. (2004). Optimum reconfiguration of radial distribution systems to maximize loadability, IEEE Trans. Power Systems, vol. 19, no. 1 , pp. $260-266$.

Wright, J. S. (1997). Primal-dual interior point methods. Society for Industrial and Applied Mathematics - SIAM, p. 21-45.

Whei-Min, L. e Hong-Chan, C. (1998). A new approach for distribution feeder reconfiguration for loss reduction and service restoration, IEEE Trans. Power Delivery, vol.13, no. 3, pp. 870-875. 